$63^{\text {ème }}$ Congrès de la SFCO, 03030 (2015)

DOI:10.1051/sfco/20156303030

(C) Owned by the authors, published by EDP Sciences, 2015

\title{
Péricardite aiguë d'origine dentaire
}

\author{
Hentati $\mathrm{H}^{*}$, Fdhila $\mathrm{K}^{* *}$, Toumi $\mathrm{A}^{* * *}$, Fdhila $\mathrm{W}^{* *}$, Chakroun $\mathrm{M}^{* * *}$, Maatouk $\mathrm{F}^{* * * *}$, \\ Bakhrouf A**, Selmi J* \\ * Laboratoire de recherche santé orale et réhabilitation bucco-faciale, Service de médecine et chirurgie \\ buccales, clinique universitaire de médecine dentaire, Rue Avicenne, 5000 Monastir, Tunisie \\ ** Laboratoire d'Analyses, Traitement et Valorisation des Polluants de l'Environnement et des Produits, \\ Faculté de Pharmacie de Monastir, Rue Avicenne, 5000 Monastir, Tunisie \\ *** Service de maladies infectieuses EPS Fattouma Bourguiba, Rue Farhat Hached, 5000 Monastir, Tunisie \\ **** Service de cardiologie EPS Fattouma Bourguiba, Rue Farhat Hached, 5000 Monastir, Tunisie
}

La péricardite aiguë est l'inflammation aiguë des deux feuillets (pariétal et viscéral) de la séreuse péricardique associée ou non à un épanchement exsudatif intrapéricardique. Elle est causée par des agents infectieux (virus, bactéries, parasites, levures) qui peuvent irriter le péricarde, soit directement, soit par le biais de la réaction immunitaire qu'ils déclenchent (Millaire 2015).

La péricardite purulente est rare mais grave. Elle touche essentiellement des patients immunodéprimés.

Un patient âgé de 28 ans, sans antécédents pathologiques notables, a été hospitalisé, en urgence, pour des douleurs thoraciques et fièvre. L'échographie a montré un décollement péricardique circonférentiel de $18 \mathrm{~mm}$ sans signes de compression avec la présence de dépôt de fibrines. Le diagnostic retenu était une péricardite aigue. L'absence d'amélioration sous traitement médical (aspégic ${ }^{\circledR}$ et colchicine ${ }^{\circledR}$ ) et le syndrome inflammatoire biologique ont redressé le diagnostic de péricardite purulente d'origine bactérienne malgré l'absence de foyer infectieux de voisinage ou d'une porte d'entrée évidente, la négativité des bilans biologiques spécifiques et les hémocultures non concluantes. Une antibiothérapie a été démarrée mais l'évolution n'était pas satisfaisante.

La recherche de foyer infectieux dentaire primaire pouvant expliquer une infection focale a été demandée.

L'examen clinique bucco-dentaire complété par un examen radiologique standard (cliché panoramique et rétro-alvéolaire de la deuxième molaire maxillaire gauche) a montré que cette dernière présentait une obturation canalaire incomplète avec une parodontite apicale chronique évoquant un granulome en rapport avec la racine disto-vestibulaire.

Tenant compte de la gravité de l'atteinte cardiaque, la décision thérapeutique était radicale par avulsion de cette dent. L’évolution était rapidement favorable.

Le profil microbiologique des bactéries du granulome était le même que celui de l'hémoculture: code 1 : stahylococcus sciuri $97,8 \%$ et staphylococcus aureus $0,7 \%$; code 2 : staphylococcus schleiferi 99,3\% et staphylococcuscapitis 0,5\% par l’Api Staph.

Les résultats par la technique PCR (polymerase chain reaction) ont montré que toutes les souches isolées présentaient des gènes codant la coagulase confirmant qu'il s'agit d'une seule souche staphylococcus aureus.

Après culture, dans un bouillon nutritif durant trois mois, toutes les souches ont repris un profil typique de staphylococcus aureus.

This is an Open Access article distributed under the terms of the Creative Commons Attribution License 4.0, which permits unrestricted use, distribution, and reproduction in any medium, provided the original work is properly cited. 
Ce cas clinique peut être classé dans le cadre d'infection focale d'origine dentaire.

La théorie de l'infection focale, présentée dès le début du $20^{\text {ème }}$ siècle (Hunter 1900), postule que des maladies systémiques peuvent être causées par des microorganismes provenant d'un foyer infectieux qui est souvent asymptomatique (Brzewski et al. 2013).

Dans des conditions de stress, staphylococcus aureus, comme d'autres bactéries, pourrait décroitre et il pourrait perdre certaines de ses activités enzymatiques aboutissant à des bactéries non cultivables (Lagha et al. 2012, Mahdhi et al. 2012).

\section{Nom et adresse du conférencier}

\section{Hajer HENTATI}

Laboratoire de recherche santé orale et réhabilitation bucco-faciale

Service de médecine et chirurgie buccales, clinique universitaire de médecine dentaire

Rue Avicenne

5000 Monastir (Tunisie)

hajer_hentati@yahoo.fr 